\title{
Analytical Derivation of Hypothetical Tumor Internal Pressure Using Physically Based Formalism
}

\author{
A. Ghanbari $^{a}$, R. Khordad ${ }^{a, *}$ And M. Ghaderi-Zefrehei ${ }^{b}$ \\ ${ }^{a}$ Department of Physics, Yasouj University, Yasouj 75918, Iran \\ ${ }^{b}$ Department of Animal Genetics, Yasouj University, Yasouj 75918, Iran
}

Received: 14.07.2020 \& Accepted: 17.08.2020

\begin{abstract}
Addressing the open, complex, dynamic and adaptive nature of cancer biology, mathematical formalism that works well at a tumor level is required. Our main goal in this study is to propose such simple mathematical formalism. We try to simplify the model as much as possible, however to such extent as not to leave out the crucial features of the molecular biology or cell physiology. Depending on the variation of the analyzed real data, the tumor physically based formalism allows for insights at the molecular level and for making robust reliable predictions about how a tumor responds to a variety of natural situations and interventions. In this study, by adopting a hypothetical cylindrical tumor shape surrounded in a hypothetical cylindrical-like peripheral tissue, a set of physically based ordinary differential equations (ODEs) was developed to assess the internal pressure of the tumor in the course of a two-dimensional external pressure. The calculation was analytically performed and applied on real data. It turned out that under constant external pressure exerted on a tumor wall, the internal fluid tumor pressure decreased over time. This study, although purely mathematical and abstract in nature, may help further studies on environmental pressures which aim at either deterring or alleviating tumor growth. Researchers should intensify efforts aimed at finding a tissue-specific physically based mathematical model to discover the underlying processes governing cancer formation and development.
\end{abstract}

topics: tumor, hypothetical, internal pressure, ODEs

\section{Introduction}

Decades ago we have witnessed progress in cancer research due to theoretical and experimental contributions from different fields of science [1-10]. Cancer cells arise from abnormal cellular growth [10]. These cells may be either benign — when localized in a certain place - or malign and metastatic, when they invasively spread inside the body through blood or lymphatic vessels. Such a theory is derived from observations of chromosomal abnormalities in cancer made in the early 20th century by Bover 1903 [11]. As seen in Fig. 1, cancer cells are organized hierarchically and at the top of this hierarchy, the cancer stem cells (CSCs) occur [12]. Therefore, the CSC hypothesis is the base for explaining tumor formation.

As mentioned above, malignant tumors can spread through the body using blood or the lymphatic system. This process is called metastasis and considered a multistep process [13]. During metastasis, tumors must overcome several obstacles when they move through the tissue. For example, they form different shapes and mechanical stiffness, and they resist shear and compression stresses

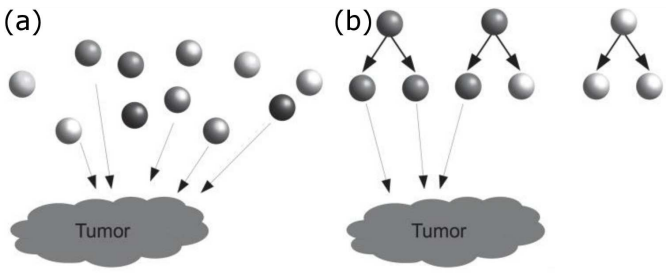

Fig. 1. (a) The stochastic model states that cancer cells are heterogeneous but all of them are tumorigenic. (b) According to the CSC hypothesis, only a subset of cells is tumorigenic. CSCs can divide symmetrically, giving rise to two CSCs or asymmetrically, giving rise to one CSC and one more differentiated cancer cell. Those cells are not tumorigenic and cannot produce CSCs [10].

from normal tissues that surround them. Their mechanical behavior under a small strain compression can be modeled using poroelastic models [14-16]. It is known that shear and stresses exert pressure on tumors in different ways. Armstrong [17] and Berry et al. [18] have derived a full set of expressions for strains and fluid pressure in uniform cylindrical samples compressed from the top. A major 
limitation in these models is the assumption that the sample is uniform and the permeability outside the uniform cylindrical sample is higher than that inside the sample.

Apart from the limitation appearing due to the sample uniformity, a major assumption in these models is that the permeability outside the uniform cylindrical sample is higher than that inside the sample. However, this is not always true for the case of a tumor surrounded by normal tissue. In many cases, vascular and interstitial permeability of tumors is higher than that of normal tissue [19]. We use this observation to study fluid transport and fluid pressure inside tumors.

Islam et al. [19] have obtained an analytical expression for displacement, strains and fluid pressure of a cylindrical poroelastic inclusion embedded in a cylindrical poroelastic background. In that model, permeability inside tumors is higher than outside. Baxter and Jain $[9,20,21]$ have described stresses, strains and fluid pressure behavior of a tumor without considering the time. In cylindrical tumors, three different stresses are exerted: an axial stress that is parallel to the axis of cylindrical symmetry, a circumferential stress that is in the tangential direction and a radial stress that is perpendicular to the axis.

In a muscle-based tissue, a tumor's response to stress contains an active component due to an actomyosin-driven contraction of the cytoskeleton. This behavior can be described theoretically with models of active fluids and gels [22-26]. In the active gel theory, the stress tensor obeys the viscoelastic constitutive equation. This theory plays an important role in explaining the mechanical behavior of tumors. Many mathematical models have been developed to represent some aspects of cancers like simulating the growth of a tumor volume [27-30]. Stress affects the fluid inside tumors and this interstitial fluid inside tumors is an interesting phenomenon in a biological process. It helps bring oxygen and nutrients to cells and remove waste products from them. When we study the interaction between the fluid flow and solid deformation within a linear porous medium, we obtain poroelasticity. The interstitial fluid pressure (IFP) is an important parameter in tumor prognosis, tumor treatment, drug delivery, tumor therapy and tumor metastasis [31-36]. Experimental and theoretical papers report that the IFP is uniform throughout the central area of a tumor [1, 9, 37-39]. Zakariapour et al. [40] have developed a numerical simulation of the interstitial fluid flow and blood flow to a tissue containing two-dimensional cylindrical tumors. They have calculated the IFP, velocity and blood pressure. Soltani and Chen [41] have developed a mathematical model of the interstitial fluid flow to the physiological system containing solid spherical tumors. Since the spherical shape of tumors has already been studied, we intend to investigate the cylindricalshape of tumors.
In this paper, we have studied the effect of an axial and circumferential stress that is exerted on cylindrical tumors. We calculated the mechanical behavior of tumors, such as pressure versus time and the effect of the Poisson ratio (EPR).

\section{Theoretical background}

There are many theoretical frameworks that can be used when modeling cells. In general, a tumor surrounded by normal tissue can be treated as a poroelastic inclusion embedded inside a poroelastic background material with different properties [42-47]. Throughout this paper, a cylindrical polar coordinate system is used for description. The pressure exerted on an axial and circumferential axis and the inclusion are assumed to be typical of the homogeneous poroelastic material.

The physics description of the mixture of a solid and a fluid constituent is based on [48]:

$$
\begin{aligned}
& \nabla\left(\boldsymbol{v}_{s}+\boldsymbol{w}_{r}\right)=0 \\
& \boldsymbol{w}_{r}=-k \nabla p, \\
& -\boldsymbol{\nabla}+\nabla \boldsymbol{\sigma}_{e}=0 .
\end{aligned}
$$

Here, $\boldsymbol{v}_{s}$ is the solid matrix velocity, $\boldsymbol{w}_{r}$ is the volumetric flux of fluid relative to the solid, $p$ is the fluid pressure, $\boldsymbol{\sigma}_{e}$ is the effective (or elastic) stress, and $k$ is the hydraulic permeability of the solid matrix. The conservation of mass relation for the mixture is given by (1). A consequence of the conservation of momentum for the fluid constituent is expressed by (2) and it reduces to Darcy's law. In turn, (3) shows the conservation of momentum for the mixture. For a linear isotropic elastic solid matrix, we have

$$
\boldsymbol{\sigma}_{\boldsymbol{e}}=\lambda_{e} \operatorname{Tr}(\boldsymbol{E}) \boldsymbol{I}+2 \mu_{s} \boldsymbol{E},
$$

where $\boldsymbol{E}$ is the infinitesimal strain tensor and $\lambda_{s}$ and $\mu_{s}$ are the Lamé constants of the solid matrix. The strain is related to the solid matrix displacement $\boldsymbol{u}$ through $\boldsymbol{E}=\left(\nabla \boldsymbol{u}+\nabla^{\mathrm{T}} \boldsymbol{u}\right) / 2$, whereas the solid velocity is given by $\boldsymbol{v}_{s}=\frac{\partial \boldsymbol{u}}{\partial t}$, where $\frac{\partial \boldsymbol{u}}{\partial t}$ represents the material derivative with respect to the solid matrix.

The above equations from the mixture theory can easily be reduced to the case of a membrane [48]. The fluid flux normal to a membrane of the unit outward normal is given by

$$
w_{n}=\boldsymbol{w} \cdot \boldsymbol{n}=-k_{m}(\nabla p) \cdot \boldsymbol{n},
$$

where $k_{m}$ is the membrane hydraulic permeability. For a thin membrane of thickness $h_{m}$, the pressure gradient normal to the membrane is given by

$$
-\nabla p \cdot \boldsymbol{n} \simeq \frac{\Delta p}{h_{m}},
$$

where $\Delta p=p(x)-p\left(x+h_{m}\right)$ is the upstream-todownstream pressure difference and represents the coordinate direction along $\boldsymbol{n}$. Thus,

$$
w_{n}=L_{p} \Delta p,
$$

where $L_{p}=k_{m} / h_{m}$ is the membrane hydraulic conductivity. 


\subsection{Mathematical aspects of poroelastic material}

Let us suppose that the inclusion $i$ with a radius is embedded in the background with radius $b$. This can be described as follows

$$
w_{r, b}=-k_{b} \frac{\partial p_{b}}{\partial r}
$$

where $w_{r, b}$ is the normal component of fluid flux in the background region and $k_{b}$ is the background permeability. The thickness of the poroelastic material is $h=b-a$ and $a$ and $b$ are radii of the inclusion and background, respectively (see Fig. 2). As the pressure acts on it, we can write

$$
w_{r, b}=-L_{p} \Delta p_{b}
$$

where $L_{p}=\frac{k_{b}}{h}$.

Under unconfined compression, the governing equations for the poroelastic sample inside the inclusion are as follow

$$
\begin{aligned}
& \frac{1}{r} \frac{\partial}{\partial r}\left[r\left(\frac{\partial \boldsymbol{u}}{\partial t}+w_{r}\right)\right]+\frac{1}{r} \frac{\partial \boldsymbol{u}}{\partial r}-\dot{\varepsilon}_{\theta}=0 \\
& w_{r, i}=-k_{i} \frac{\partial p_{i}}{\partial r} \\
& -\frac{\partial p}{\partial r}+\lambda\left[\frac{1}{r} \frac{\partial}{\partial r}\left(r \frac{\partial u_{r}}{\partial r}\right)+\frac{1}{r^{2}} \frac{\partial^{2} u_{\theta}}{\partial \theta^{2}}\right] \\
& +\frac{2 \mu}{r} \frac{\partial}{\partial r}\left(r \frac{\partial u_{r}}{\partial r}\right)=0 .
\end{aligned}
$$

Integrating (10) with respect to $r$ and making use of the fact that there is no displacement along the center line of the system, we obtain

$$
w_{r, i}=-2 \frac{\partial u_{i}}{\partial t}+\frac{r}{2} \dot{\varepsilon}_{\theta} .
$$

Next, combining (11)-(13), a partial differential equation for the radial displacement becomes

$$
\begin{aligned}
& a L_{p}(\lambda+2 \mu)\left(\frac{\partial^{2} u_{i}}{\partial r^{2}}+\frac{1}{r} \frac{\partial u_{i}}{\partial r}\right) \\
& +\frac{a L_{p} \lambda}{r^{2}} \frac{\partial^{2} u_{\theta}}{\partial \theta^{2}}+\frac{2 a L_{p}}{k_{i}} \frac{\partial u_{i}}{\partial t}+\frac{a L_{p}}{2 k_{i}} r \dot{\varepsilon}_{\theta}=0 .
\end{aligned}
$$

Here, we consider the case that permeability of the inclusion is much higher than the permeability of the background, $\frac{a L_{p}}{k_{i}} \gg 1$ [48]. Thus, neglecting the terms with $\frac{a L_{p}}{k_{i}}$, we obtain

$$
\frac{\partial}{\partial r}\left(\frac{\partial u}{\partial r}+\frac{u}{r}\right)=0
$$

\subsection{Inside the inclusion calculation}

When integrating (15) with respect to $r$, one gets

$$
u_{i}(t)=r \varepsilon_{r r, i}(t) \text {, }
$$

where $\varepsilon_{r r, i}(t)$ - the radial normal strain inside the inclusion. Note that the function $\varepsilon_{r r, i}(t)$ in this solution is uniform. As a result, also the fluid pressure inside the inclusion becomes independent of the radius and constant with respect to the radius,

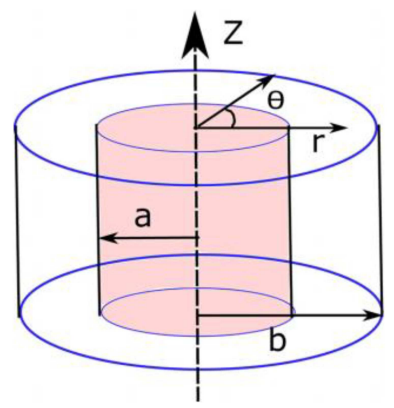

Fig. 2. A cylindrical sample of a poroelastic material of radius $b$ with a cylindrical inclusion of radius $a$. Axial direction is along the $z$ direction, radial direction is along the $r$ direction and the circumferential direction is along the $\theta$ angle.

i.e., $p_{i}(r, t)=p_{i}(t)$. The fluid pressure can be obtained from the elastic stress, so according to the exerted circumferential stress one has

$$
\begin{gathered}
-p(a, t)+\lambda\left(\frac{\partial u_{r}}{\partial r}+\varepsilon_{\theta}\right)_{r=a} \\
+2 \mu\left(\frac{\partial u_{r}}{\partial r}+\frac{u_{r}}{r}\right)_{r=a}=0 .
\end{gathered}
$$

As the zero fluid pressure condition applies at the outer boundary and the continuity of the fluid pressure at the interface between the inclusion and the background $\left(p_{i}(a, t)=p_{b}(a, t)\right)$, then

$$
L_{p} p_{b}(a, t)=L_{p} p_{i}(a, t)=w_{r, i}(a, t)=w_{r, b}(a, t) .
$$

From (13) and (18), we deduce that

$$
L_{p} p_{i}(a, t)=\left(-2 \frac{\partial u_{r}}{\partial t}+\frac{r}{2} \dot{\epsilon}_{\theta}(t)\right)_{r=a}
$$

and when one substitutes (17), it leads to

$$
\begin{aligned}
& \frac{1}{L_{p}}\left(2 a \dot{\varepsilon}_{r r}(t)+\frac{a}{2} \dot{\varepsilon}_{\theta}(t)\right) \\
& \quad+(\lambda+2 \mu) \varepsilon_{r r}(t)+\lambda \varepsilon_{\theta}(t)=0 .
\end{aligned}
$$

Furthermore,

$$
p(t)=(\lambda+2 \mu) \varepsilon_{r r}(t)+\lambda \varepsilon_{\theta}(t)
$$

because the fluid pressure does not depend on the radial position, $p_{i}(t)=p_{i}(a, t)$. All this results in

$$
\begin{gathered}
2 a \dot{\varepsilon}_{r r}(t)+L_{p}(\lambda+2 \mu) \varepsilon_{r r}(t) \\
+\lambda L_{p} \varepsilon_{\theta}(t)+\frac{a}{2} \dot{\varepsilon}_{\theta}(t)=0 .
\end{gathered}
$$

In the case of stress relaxation, it responds to a strain step accordingly $\varepsilon_{\theta, i}(t)=-\varepsilon_{0} H(t)$, where $\varepsilon_{0}$ is the magnitude strength and $H(t)$ is the Heaviside step function. Therefore, $(22)$ reduces to the form

$$
2 a \dot{\varepsilon}_{r r}(t)+L_{p}(\lambda+2 \mu) \varepsilon_{r r}(t)+\lambda L_{p} \varepsilon_{0}=0
$$

with solution

$$
\varepsilon_{r r}(t)=\frac{\lambda L_{p} \varepsilon_{0}+\exp \left(\frac{L_{p}(\lambda+2 \mu)}{2 r} t\right)}{L_{p}(\lambda+2 \mu)} .
$$


The equation for the EPR inside the inclusion is given as

$$
\Omega_{i}(t)=\frac{\varepsilon_{r r, i}(t)}{\varepsilon_{0}}=\frac{\lambda L_{p} \varepsilon_{0}+\exp \left(-\frac{L_{p}(\lambda+2 \mu)}{2 r} t\right)}{L_{p} \varepsilon_{0}(\lambda+2 \mu)},
$$

for the fluid pressure as

$$
p_{i}(r, t)=\frac{\lambda L_{p} \varepsilon_{0}+\exp \left(-\frac{L_{p}(\lambda+2 \mu)}{2 r} t\right)}{L_{p}}+\lambda,
$$

and for the radial displacement inside the inclusion (deduced from (16)) as

$$
u_{i}(r, t)=\frac{\lambda L_{p} \varepsilon_{0}+\exp \left(\frac{L_{p}(\lambda+2 \mu)}{2 r} t\right)}{L_{p}(\lambda+2 \mu)} r .
$$

\subsection{Outside the inclusion calculation}

The continuity equation of the pore fluid is a basic poroelastic equation for cylindrical symmetry which reads as [49]

$$
\frac{\partial \varepsilon_{b}}{\partial t}=k_{b}\left(\frac{\partial^{2} p_{b}}{\partial r^{2}}+\frac{1}{r} \frac{\partial p_{b}}{\partial r}\right)
$$

To express the equation of equilibrium in terms of volumetric strain, we use the volumetric strain that is related to the radial displacement. Hence

$$
\varepsilon_{b}=\frac{\partial u}{\partial r}+\frac{u}{r}+\varepsilon_{\theta}=\frac{1}{r} \frac{\partial(r u)}{\partial r} .
$$

and (28) takes the following form [49]

$$
\frac{\partial \sigma_{r r}}{\partial r}+\frac{\sigma_{r r}-\sigma_{\theta, \theta}}{r}=0
$$

where $\sigma_{r r}$ and $\sigma_{\theta, \theta}$ are stresses in radial and circumferential (tangential) directions, respectively. These stresses can be separated into the elastic stresses and the fluid pressure

$$
\sigma_{r r}=\sigma_{r r}^{\prime}+p, \quad \sigma_{\theta, \theta}=\sigma_{\theta, \theta}^{\prime}+p,
$$

while the general character of the equilibrium equation remains unchanged, i.e.,

$$
\frac{\partial \sigma_{r r}^{\prime}}{\partial r}+\frac{\sigma_{r r^{\prime}}-\sigma_{\theta, \theta}^{\prime}}{r}=0
$$

Now, we will use (29) and the stress-strain dependence to obtain

$$
\begin{aligned}
& \sigma_{r r}^{\prime}=-\left(K-\frac{2}{3} G\right) \varepsilon_{b}-2 G \frac{\partial u}{\partial r} \\
& \sigma_{\theta, \theta}^{\prime}=-\left(K-\frac{2}{3} G\right) \varepsilon_{b}-2 G \frac{u}{r}
\end{aligned}
$$

with the help of which a simpler version of the equation of equilibrium will be available, namely

$$
\left(K+\frac{4}{3} G\right) \frac{\partial \varepsilon_{b}}{\partial r}=\frac{\partial p_{b}}{\partial r} .
$$

Here, $K$ is the bulk modulus and $G$ is the shear modulus of the porous medium.

Let us assume that $H_{A b}(t)=K+\frac{4}{3} G$ and integrate (35) with respect to $r$. As a result, $\varepsilon_{b}=\frac{p}{H_{A b}}+Q_{b}$ and $Q_{b}$ is the integrating constant.
When substituting the resulted function into (28), we obtain

$$
\frac{\partial^{2} p_{b}}{\partial^{2} r}+\frac{1}{r} \frac{\partial p_{b}}{\partial r}=\frac{1}{c_{b}} \frac{p_{b}}{t}+\frac{1}{c_{b}} \frac{\partial Q_{b}}{\partial t},
$$

where $c_{b}=H_{A b} k_{b}$ is the gel diffusion [50].

For an applied strain step magnitude $\varepsilon_{0}$ along the negative tangential direction, the boundary condition of the fluid pressure outside the inclusion is $p_{b}(r, t)=p_{i}(t)$ at $r=a$, and $p_{b}(r, t)=0$ at $r=b$. The initial condition for this pressure at $t=0$ is $p_{b}(r, t)=\mu_{b} \varepsilon_{0}$.

We now divide our solution in two distinct parts. In the first case, (36) is considered for the initial condition when the zero fluid pressure at the interface of the inclusion and background occurs. Then, the solution at $r=a$ and $r=b$ becomes [50]

$p_{b, 1}=\mu_{b} \varepsilon_{0} \pi \sum_{n=1}^{\infty} \frac{J_{0}\left(\beta_{n} a\right) U\left(\beta_{n} r\right)}{J_{0}\left(\beta_{n} a\right)+J_{0}\left(\beta_{n} b\right)} \mathrm{e}^{-\beta_{n}^{2} c_{b} t}$

where $U\left(\beta_{n} r\right)=J_{0}\left(\beta_{n} r\right) Y_{0}\left(\beta_{n} b\right)-Y_{0}\left(\beta_{n} r\right) J_{0}\left(\beta_{n} b\right)$ and $\beta_{n}$ 's are the roots of the function $C_{1}(x)=$ $J_{0}(x a) Y_{0}(x b)-Y_{0}(x a) J_{0}(x b)=0$.

In the second case, (36) is solved for the boundary condition at the interface imposed by the fluid pressure inside the inclusion, $p_{b}(a, t)=p_{i}(a, t)$. The solution to the second part of the problem can be written as follows [51]

$$
\begin{gathered}
p_{b, 2}=-\mu_{i} \varepsilon_{0} \pi \sum_{n=1}^{\infty} \frac{J_{0}\left(\beta_{n} a\right) J_{0}\left(\beta_{n} b\right)}{J_{0}^{2}\left(\beta_{n} a\right)-J_{0}^{2}\left(\beta_{n} b\right)} \\
\times \frac{\mathrm{e}^{-t / \tau_{\varepsilon_{i}}}-\mathrm{e}^{\beta_{n}^{2} c_{b} t}}{\beta_{n}^{2}-1 /\left(\tau_{\varepsilon_{i}} c_{b}\right)} \beta_{n}^{2} U\left(\beta_{n} r\right)
\end{gathered}
$$

The total pressure can be written as

$$
\begin{aligned}
& p_{b}(r, t)=-\mu_{i} \varepsilon_{0} \pi \sum_{n=1}^{\infty} \frac{J_{0}\left(\beta_{n} a\right) J_{0}\left(\beta_{n} b\right)}{J_{0}^{2}\left(\beta_{n} a\right)-J_{0}^{2}\left(\beta_{n} b\right)} \\
& \times \frac{\mathrm{e}^{-t / \tau_{\varepsilon_{i}}}-\mathrm{e}^{\beta_{n}^{2} c_{b} t}}{\beta_{n}^{2}-1 /\left(\tau_{\varepsilon_{i}} c_{b}\right)} \beta_{n}^{2} U_{0}\left(\beta_{n} r\right) \\
& +\mu_{b} \varepsilon_{0} \pi \sum_{n=1}^{\infty} \frac{J_{0}\left(\beta_{n} a\right) U\left(\beta_{n} r\right)}{J_{0}\left(\beta_{n} a\right)+J_{0}\left(\beta_{n} b\right)} \mathrm{e}^{-\beta_{n}^{2} c_{b} t} .
\end{aligned}
$$

By considering the stress at $a<r<b$ equal to zero, we can write

$$
p_{b}(r, t)=\lambda_{b}\left(\frac{\partial u_{b}}{\partial r}-\varepsilon_{0}\right)+2 \mu\left(\frac{\partial u_{b}}{\partial r}+\frac{u_{b}}{r}\right) .
$$

Taking the general displacement as

$$
u_{b}(r, t)=r \varepsilon_{r r, b}(r, t),
$$

just as the same derivation that was done for the radial strain inside the inclusion, the radial strain in the background region is given by

$$
\varepsilon_{r r, b}(r, t)=\frac{p_{b}(r, t)+\lambda_{b} \varepsilon_{0}}{\lambda_{b}+4 \mu_{b}} .
$$

We can express the EPR equation for the background region as follows

$$
\Omega_{b}(t)=\frac{\varepsilon_{r r, b}(t)}{\varepsilon_{0}}=\frac{p_{b}(r, t)+\lambda_{b} \varepsilon_{0}}{\varepsilon_{b}\left(\lambda_{b}+4 \mu_{b}\right)} .
$$




\section{Results and discussion}

In this section, using a proposed model, we carried out our calculations to measure the EPR parameters and pressure values of the three samples obtained from Tauhidal [19]. To get an instantaneous response of the pressure and EPR parameter inside and outside the tumors, we used the relations in (25), (26), (39) and (43). We exerted strain in such a way that instantaneous values of radial and circumferential stresses inside the samples were considered. However, the effective stress on the solid phase of the poroelestic samples is not zero which initiates the recoiling of the solid phase. Therefore, as the solid matrix was completely fixed, the EPR of materials became the drained Poisson ratio which can be deduced by considering $t \rightarrow \infty$ in (25) and (43). According to (26) and (39), the fluid pressure becomes zero if we let $t \rightarrow \infty$. Based on these observations, we can conclude that at $t=0^{+}$, the solid matrix in both the inclusion and background behaves like an incompressible solid. Figure 3 shows the EPR versus time for the three samples. The EPR (in) and EPR (out) correspond to the inside and the background of the solution, respectively. Figure 3 demonstrates that the curves start from different values at $t=0$ for the inside and the background. Also, the EPR for all the samples is reduced with increasing the time until it reaches a fixed value (the drained Poisson's ratio). The drained Poisson's ratio depends on the material properties of the sample. The change of the EPR with time for all the studied samples shows that the considered tumors are not incompressible materials. The reason for the difference between the $\mathrm{EPR}$ at $t=0$ is the difference between permeability of the inclusion and the background.

In each curve, the permeability of the inclusion and the background is different as the inclusion in sample A has 1000 times higher interstitial permeability than the background, the inclusion in sample B has 50 times higher interstitial permeability than the background and the inclusion in sample $\mathrm{C}$ has 20 times higher interstitial permeability than the background.

Figure 4 shows the fluid pressure versus time for all the samples inside and the background of the solution from the analytical model. Here, we have compared the fluid pressure from the inside and the outside of the inclusion. The curves' behavior shows that the fluid pressure of the inclusion and the background has the same manner with time. The inside and outside pressures are reduced with passing time for all the samples. Also, we observe that the fluid pressure goes to zero at steady-state for all the samples. The pressure of the inside is higher than the outside pressure at $t=0$ because the permeability of the inside is higher than the outside.

Furthermore, in Fig. 5, we show the pressure as a function of time for different radii of the cylinder. It can also be observed that the pressure

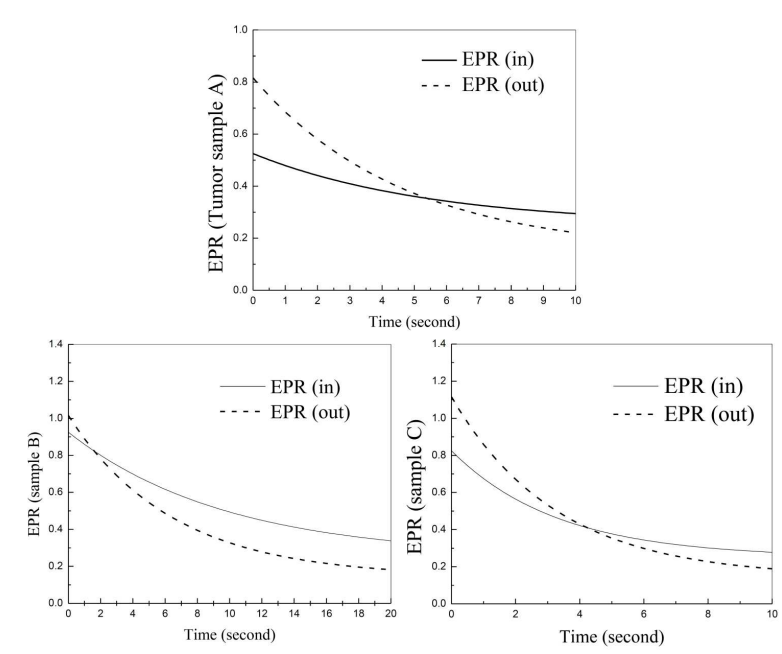

Fig. 3. EPR versus time for three samples.

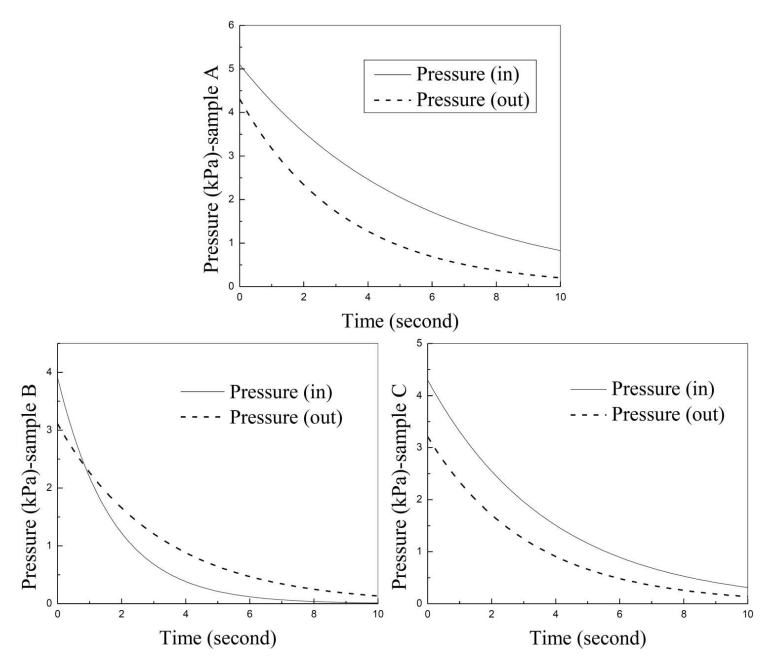

Fig. 4. Fluid pressure versus time for all samples inside and the background of solution from the analytical model.

in the inclusion decreases with increasing the time for different radii because the system approaches to the steady state. Also, at a fixed time, the pressure increases with the radius because the permeability of the inclusion is higher than that of the background. For a higher radius, the pressure tends to zero.

This model could become a useful tool for extracting the material properties of tumors and surrounding tissues and can be applied for axial and lateral strains estimated from the pre- and postcompression radio frequency data to obtain clinically important parameters such as the Poisson ratio, the product of interstitial permeability and aggregate modulus of the tumor and normal tissues and other relevant mechanical parameters [18]. In this work, we cannot use only the simulation methods because of the large number of parameters involved in the model and because of the effect of boundary conditions on the displacement and strain 


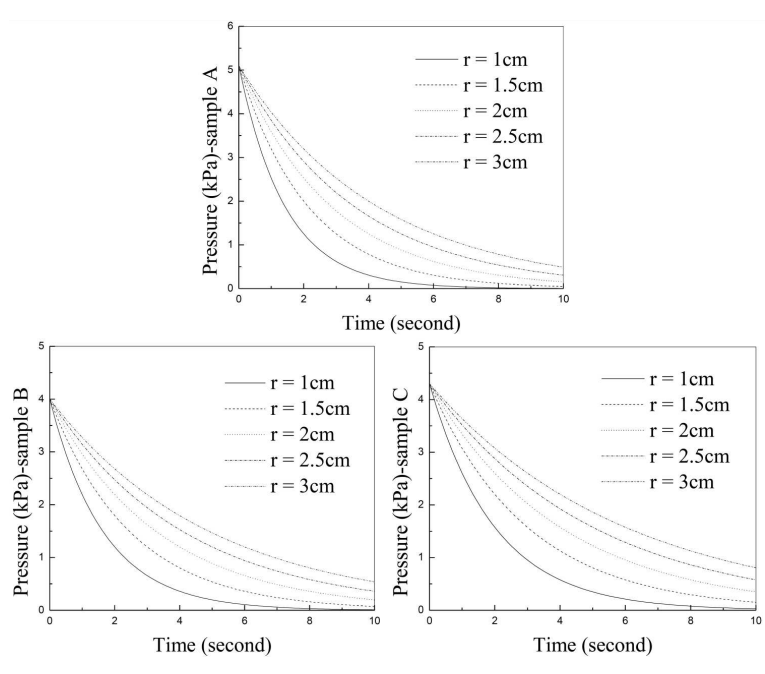

Fig. 5. Pressure versus time for different cylindrical samples with different radiuses.

data. According to the time-pressure diagrams, the pressure on the inner walls is first calculated which drops to zero after a period of time. This may indicate that after the lapse of time $t$, the walls of the tumor have ruptured and there are no more walls that force fluid into the tumor. Thus, as the tumor wall ruptures, the fluid exits and is transported to other parts of the body through blood or lymphatic vessels. This factor may be the onset of metastasis based upon our assumptions in this study.

\section{Conclusion}

We have analytically investigated the displacement, strains and fluid pressure for the cylindrical poroelastic inclusion embedded in the cylindrical poroelastic background using the theoretical model. This model helps to better understand the mechanical behavior of tumors. We have studied the radial and lateral strain exerted on tumors and showed that the metastasis-like phenomenon could be triggered at a special time. Also, we have derived models focusing on ultrasound poroelastography application. Residing in a complex microenvironments, tumor cells are exposed and subjected to a wide variety of physical and chemical stimuli. This would influence cell behavior to either progress or inhibit the tumor. It is our responsibility - as pure mathematical and biophysicsminded modelers - to create physiological models that enable accurate understanding of the multidimensional structure, organization and complex relationships in diverse tumor microenvironments. This sort of models can greatly expedite clinical discovery by closely replicating the physiological conditions while maintaining high tunability and control of the extrinsic factors. In this study, we tried to come up with new models that target key aspects of the tumor surrounding tissues and their role in cancer progression.

\section{References}

[1] M. Sarntinoranont, F. Rooney, M. Ferrari, Ann. Biomed. Engin. 31, 327 (2003).

[2] E.A. Swabb, J. Wei, P.M. Gullino, Cancer Res. 34, 2814 (1974).

[3] P.A. Netti, L.T. Baxter, Y. Boucher, R. Skalak, R.K. Jain, Cancer Res. 55, 5451 (1995).

[4] I. Karpiel, Z. Drzazga, P. Mazgaj, M. Drzazga, A. Giec-Lorenz, Acta Phys. Pol. A 133, 728 (2018).

[5] R.K. Jain, J.D. Martin, T. Stylianopoulos, Ann. Rev. Biomed. Engin. 16, 321 (2014).

[6] R.K. Jain, R.T. Tong, L.L. Munn, Cancer Res. 67, 2729 (2007).

[7] P.A. Netti, L.T. Baxter, Y. Boucher, R. Skalak, R.K. Jain, AIChE J. 43, 818 (1997).

[8] L.T. Baxter, R.K. Jain, Microv. Res. 41, 252 (1991).

[9] L.T. Baxter, R.K. Jain, Microv. Res. 37, 77 (1989).

[10] D. Wodarz, N.L. Komarova, Dynamics of Cancer Mathematical Foundations of Oncology (2014).

[11] R. Abolfath, Y. Helo, L. Bronk, A. Carabe, D. Grosshans, R. Mohan, Eur. Phys. J. D 73, 64 (2019).

[12] D. Bonnet, J.E. Dick, Nature Med. 3, 730 (1997).

[13] C.A. Klein, Science 321, 1785 (2008).

[14] M.A. Biot, J. Appl. Phys. 12, 155 (1941).

[15] A. Malandrino, E. Moeendarbary, Poroelasticity of Living Tissues, Elsevier (2019).

[16] M.T. Islam, A. Chaudhry, G. Unnikrishnan, J.N. Reddy, R.Righetti, Phys. Med. Biol. 63, 025031 (2018).

[17] C. Armstrong, W. Lai, V. Mow, J. Biomech. Engin. 106, 165 (1984).

[18] G.P. Berry, J.C. Bamber, C.G. Armstrong, N.R. Miller, P.E. Barbone, Ultrasound Med. Biol. 32, 547 (2006).

[19] M.T. Islam, A. Chaudhry, G. Unnikrishnan, J. Reddy, R. Righetti, J. Engin. Sci. Med. Diagnos. Therap 1, 15 (2018).

[20] L.T. Baxter, R.K. Jain, Microv. Res. 40, 246 (1990).

[21] R.K. Jain, L.T. Baxter, Cancer Res. 48, 7022 (1988).

[22] T.B. Liverpool, M.C. Marchetti, Phys. Rev. Lett. 90, 138102 (2003).

[23] J.F. Joanny, J. Prost, HFSP J. 3, 94 (2009). 
[24] R. Voituriez, J.F. Joanny, J. Prost, Europhys. Lett. 70, 404 (2005).

[25] S. Prost, F. Relouzat, M. Spentchian et al., Nature 525, 380 (2015).

[26] M.C. Marchetti, J.F. Joanny, S. Ramaswamy, T.B. Liverpool, J. Prost, M. Rao, R.A. Simha Rev. Mod. Phys. 85, 1143 (2013).

[27] Y. Kim, M.A. Stolarska, H.G. Othmer, Math. Model. Method. Appl. Sci. 17, 1773 (2007).

[28] V. Cristini, X. Li, J.S. Lowengrub, S.M. Wise, J. Math. Biol. 58, 723 (2009).

[29] T.S. Deisboeck, G.S. Stamatakos, Multiscale Cancer Modeling, CRC press, 2010.

[30] Y. Kim, M.A. Stolarska, H.G. Othmer, Prog. Biophys. Molec. Biol. 106, 353 (2011).

[31] S. Ferretti, P.R. Allegrini, M.M. Becquet, P.M. McSheehy, Neoplas. 11, 874 (2009).

[32] A.V. Salnikov, V.V. Iversen, M. Koisti, Ch. Sundberg, L. Johansson, L.B. Stuhr, M. Sjöquist, H. Ahlström, R.K. Reed, K. Rubin, The FASEB J. 17, 1756 (2003).

[33] S.J. Lunt, T.M.K. Kalliomaki, A. Brown, V.X. Yang, M. Milosevic R.P. Hill, $B M C$ Cancer 8, 2 (2008).

[34] E.K. Rofstad, E.-B.M. Ruud, B. Mathiesen K. Galappathi, Clin. Cancer Res. 16, 936 (2010).

[35] S.-G. Yeo, J.-S. Kim, M.-J. Cho, K.-H. Kim, J.-S. Kim, Clin. Cancer Res. 15, 6201 (2009).

[36] T.P.F. Gade, I.M. Buchanan, M.W. Motley, Y. Mazaheri, W.M. Spees, J.A. Koutcher, Clin. Cancer Res. 15, 247 (2009).
[37] Y. Boucher, L.T. Baxter, R.K. Jain, Cancer Res. 50, 4478 (1990).

[38] J. Pusenjak, D. Miklavcic, Simul. Pract. Theor. 8, 17 (2000).

[39] H. Wiig, E. Tveit, R. Hultborn, R.K. Reed, L. Weiss, Scand. J. Clin. Lab. Invest. 42, 159 (1982).

[40] M. Zakariapour, M.H. Hamedi, N. Fatouraee, Acta Scientiarum. Tech. 40, 17 (2018).

[41] M. Soltani, P. Chen, Plos One 6, e20344 (2011).

[42] J. Rice, J. Rudnicki, D.A. Simons, Int. J. Solid. Struct. 14, 289 (1978).

[43] Y. Song, H. Hu, J.W. Rudnicki, Int. J. Solid. Struct. 83, 154 (2016).

[44] Y. Song, H. Hu, J.W. Rudnicki, Y. Duan, Geophys. J. Int. 206, 1677 (2016).

[45] J.G. Berryman, J. Math. Phys. 26, 1408 (1985).

[46] M. Kanj, Y. Abousleiman, Int. J. Numer. Analyt. Method. Geomech. 29, 103 (2005).

[47] L. Cui, Y. Abousleiman, J. Engin. Mech. 127, 391 (2001).

[48] G.A. Ateshian, K.D. Costa, C.T. Hung, Biomech. Model. Mechanobiol. 6, 91 (2007).

[49] M. Bakker, Water Resour. Res. 52, 1705 (2016).

[50] A. Grodzinsky, V. Roth, E. Myers, W. Grossman, V. Mow, J. Biomech. Engin. 103, 221 (1981).

[51] M. Muskat, J. Appl. Phys. 8, 274 (1937). 